\title{
Hybrid Radio over Fiber and Visible Light (RoF-VLC) Communication System
}

\author{
A. M. Khalid, G. Cossu, R. Corsini, M. Presi, E. Ciaramella \\ Scuola Superiore Sant'Anna, via G. Moruzzi 1, 56124, Pisa, ITALY; \\ a.khalid@sssup.it; g.cossu@sssup.it
}

\begin{abstract}
We experimentally demonstrate the integration of Radio-over-Fiber and Visible Light Communication technologies, into a hybrid system for indoor communication. The system, realized according to IEEE $802.11 \mathrm{~g}$ standard, works effectively at typical office luminance level. OCIS codes: (060.2605) Free-space optical communication; (060.4250) Networks
\end{abstract}

\section{Introduction}

Future illumination systems will increasingly use visible light-emitting diodes (LEDs). Hence these devices could be conveniently exploited also for Visible Light Communication (VLC) in indoor wireless communication and networking [1,2]. Typically white light illumination is achieved by using either a blue LED covered with a phosphor layer or combining red, green and blue (RGB) LEDs. Although VLC has been demonstrated with both options, the RGB solution is more promising, since in the other case, the bandwidth is much smaller, because of the phosphor, or has a reduced power efficiency (if combined with blue filtering) [3-4]. In both cases, to achieve high rates, the small bandwidth of the LEDs requires spectrally efficient modulation techniques like DMT and OFDM data. Visible light communication using RGB LEDs are demonstrated, with very low data rate up to $19.2 \mathrm{kbps}$ in [5]. On the other side, the radio over fiber (RoF) technology is a competitive candidate for providing broadband data to several subscribers, with higher flexibility than in wired access [6]. In this paper we investigate the coexistence of these technologies, and we report a hybrid communication system RoF-VLC using RGB LEDs with OFDM operating at maximum limit of radio wireless standard IEEE 802.11g (54 Mbit/s). We achieved good performance in terms of signal quality and $\mathrm{Tx}-\mathrm{Rx}$ distance without any pre-equalization process as show in $[7,8]$.

\section{Experimental Setup}

The experimental scheme for both downlink and uplink is shown in figure 1, for both transmission directions, i.e. RoF-VLC and VLC-RoF. The IEEE 802.11g compliant signal was generated by means of an VSG. It had 52 subcarriers; all modulated by 64 QAM within the overall bandwidth of $20 \mathrm{MHz}$. The data rate is $54 \mathrm{Mbit} / \mathrm{s}$, e.g. high enough to broadcast real time HDTV. The RoF transmitter was implemented by a single directly modulated DFB laser at $1550 \mathrm{~nm}$. The output signal from the RoF transmitter (power: $9.6 \mathrm{dBm}$, at $1550 \mathrm{~nm}$ ) was coupled into a standard single mode fiber ( $\mathrm{SMF}, \mathrm{L}=1 \mathrm{~km})$.

A variable optical attenuator (VOA) was used to control the power at the input of the PIN photodiode (15 GHz bandwidth). The RF electrical output from the photodiode was then down converted by using a mixer and a local

(a)
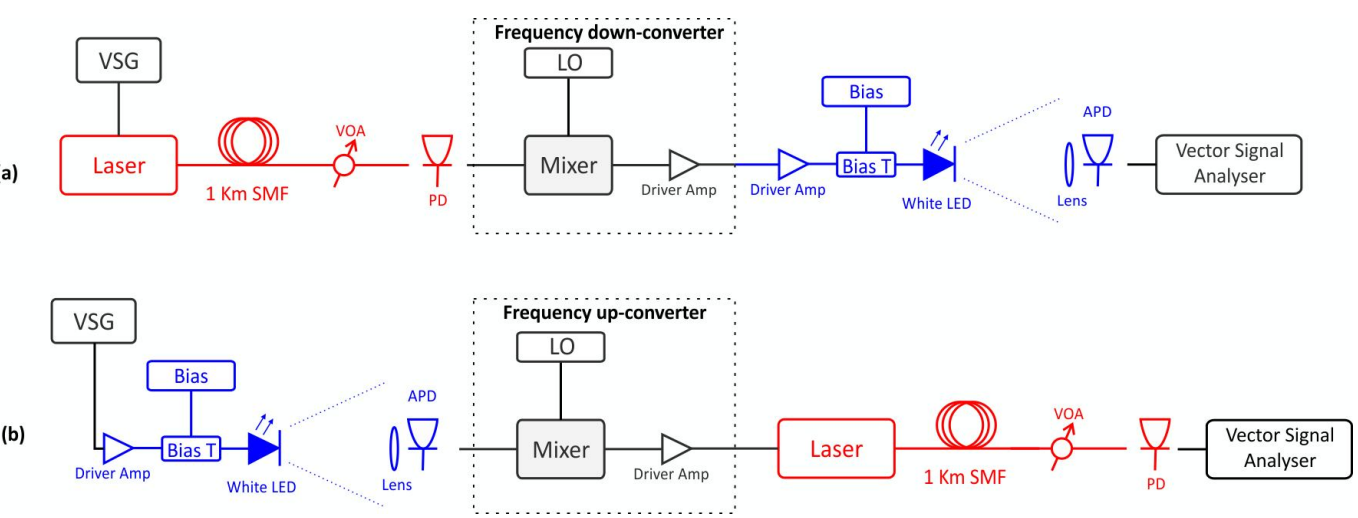

Fig. 1. Experimental scheme for downlink (a) and uplink (b) system. VSG: Vector Signal Generator. VOA: Variable Optical Attenuator. PD: Photodiode. LO: Local Oscillator. APD: Avalanche PhotoDiode. 
oscillator (fixed at the frequency $2.387 \mathrm{GHz}$ ). We thus obtained a signal centered at $25 \mathrm{MHz}$, which was electrically amplified and used to drive the LEDs. The three LEDs (Luxeon Tri-star RGB) were in a commercially available RGB LED module, devised for lightning applications. This module consists in three custom chips (one for each color), providing a total luminous flux $\sim 270 \mathrm{~lm}$ (at $700 \mathrm{~mA}$ ) and a $120^{\circ}$ full opening angle at half maximum intensity. We note that although the RF central frequency $(25 \mathrm{MHz})$ was quite outside the bandwidth of the LED (8 $\mathrm{MHz}$ ), in this frequency region the LEDs had a residual response which was enough to successfully transmit the down-converted WiFi signal.

The RF signal was then superimposed on the LEDs bias current. To achieve the best results, the RGB LEDs were operated in quasi-linear region around $400 \mathrm{~mA}$ bias current. The receiver module was placed in front of the LEDs with proper alignment to ensure the direct LOS (line-of-sight) between the Tx and Rx. The analogue receiver module consisted of a bi-convex glass lens $(25,4 \mathrm{~mm}$ focal length) and an APD module (100 MHz bandwidth, around $12 \mathrm{~A} / \mathrm{W}$ of responsivity). The light collected by the lens was concentrated on to the active area of the APD (1 mm effective diameter). The quality of the received signal was then analyzed by means of a Vector Signal Analyzer (VSA). The illumination level, which is the most relevant parameter for indoor office environment, could be changed be varying the distance between the Tx and Rx, and it was measured by a lux meter placed directly in front of the APD: in all cases it was found to be well below the standard limit of around 400-600 lux [9]. In real scenarios, arrays of LEDs could therefore be installed to ensure this desired level of luminance.

The uplink was the reverse of the downlink (see Fig. 1 b). In this second configuration, a signal at $25 \mathrm{MHz}$ frequency (the aforementioned IF) was produced and used to modulate the LED; after transmission over free space, this signal was detected by the APD. The resulting electrical signal was then up-converted to $2.412 \mathrm{GHz}$ by mixing it with a $2.387 \mathrm{GHz}$ tone generated by the local oscillator; the resulting signal was then sent to the RoF transmitter. The directly modulated optical signal was then coupled to the fiber (SMF) and received by the PIN photodiode; the quality of the electrical output of this photodiode was analyzed by a vector signal analyzer.

\section{Results and Discussions}

Since the primary function of the used LEDs is general illumination, the brightness level in front of the VLC Rx was considered as the most relevant parameter in our measurements. In order to evaluate the performance of the RoF-VLC system for a real scenario, we measured the quality of the received signal in terms of Error Vector Magnitude (EVM) for different levels of luminance, varying the VLC distance Tx-Rx from $1 \mathrm{~m}$ to $3 \mathrm{~m}$, for both downlink and uplink communications. As it is known, the performance limit given in the standard IEEE 802.11g for 64 QAM signal is EVM<5.6\% (-25 dB) [10].

We present the downlink and uplink performance for the RoF-VLC system in Fig. 2. Here we report the constellation diagrams of the 64-QAM modulated signals at $1 \mathrm{~m}$ and at $3 \mathrm{~m}$ distance between the VLC transmitter and receiver for downlink and uplink communication. Clearly, in the case of higher distance (lower luminance) we have a deterioration of signal quality (more noise at the constellation point) because the power decreases as the

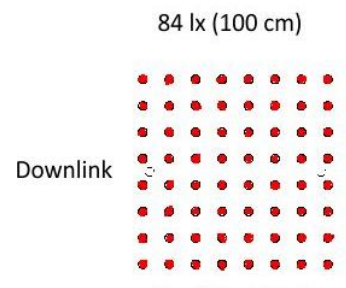

(a) $E V M=-34.7 \mathrm{~dB}$

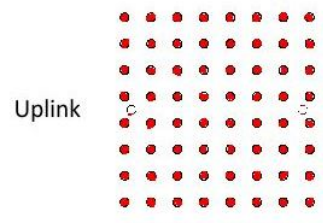

(c) $E V M=-35.6 \mathrm{~dB}$

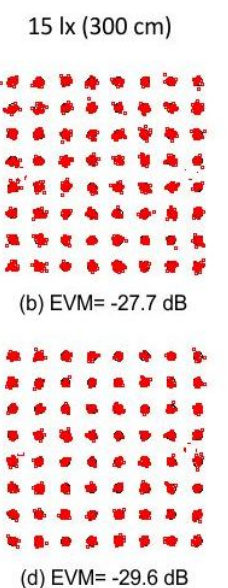

(d) $E V M=-29.6 \mathrm{~dB}$

Fig. 2. Constellation diagrams for: downlink at $100 \mathrm{~cm}$ (a), and at $300 \mathrm{~cm}$ (b) and for uplink system at $100 \mathrm{~cm} \mathrm{(c),} \mathrm{at} 300 \mathrm{~cm}$ (d) VLC distance.

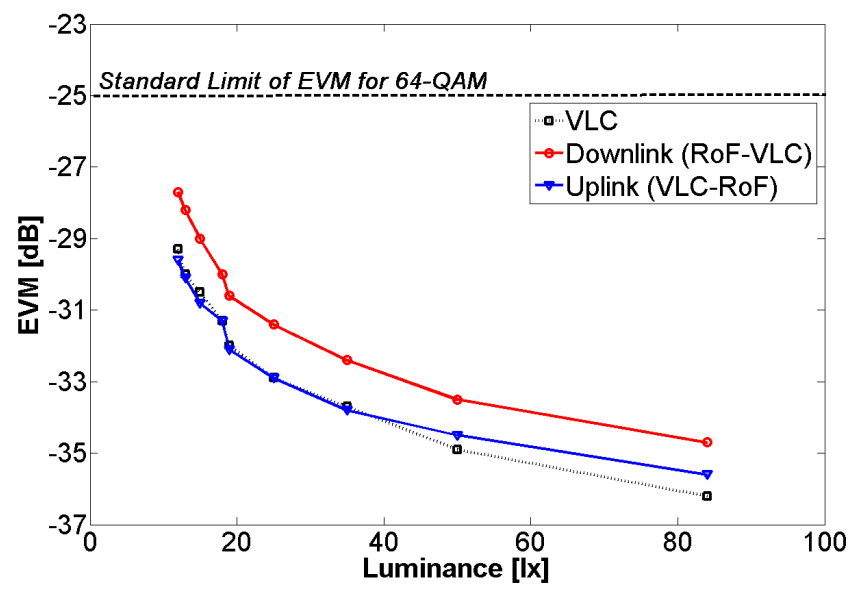

Fig. 3. System performance in terms of EVM as a function of receiver luminance for both downlink (red) and uplink (blue); luminance values are taken for a free space distance between $1 \mathrm{~m}$ and $3 \mathrm{~m}$. 
inverse of the distance squared. In the figure, as examples, we report in the first row the constellations taken for downstream, whilst in the second row we show the constellations taken for upstream communication. In both cases we report the diagrams taken for $1 \mathrm{~m}\left(1^{\text {st }}\right.$ column $)$ and $3 \mathrm{~m}\left(2^{\text {nd }}\right.$ column $)$ distance. We can see from the reported EVM values that downlink and uplink performance were similar and both slightly degraded at $3 \mathrm{~m}$ distance; however, at any distance, we typically measured a slightly better EVM value in uplink than in downlink (around 1.5 $\mathrm{dB}$ difference). This is due to the fact that in the downlink configuration the signal produced by the RoF receiver, although having an excellent EVM $(-45 \mathrm{~dB})$, has a limited electrical power, which, after mixing and amplification, leads to a lower SNR.

In Fig. 3, we summarize the system performance in terms of EVM values for both downlink and uplink configurations as a function of the illumination (red circles: downlink; blue triangles: uplink). In the same figure we also show the maximum performance that we obtained by transmitting the same signal only over the VLC (dashed line with square markers): this is limited by low LEDs frequency response. The data were measured by moving the VLC RX away from the TX with $25 \mathrm{~cm}$ step distance, and readings were taken from the VSA. From the Fig. 3 it is clear that the uplink signal is mainly limited by the VLC part of the system, whereas the downlink signal was also affected by an additional penalty, as explained before. However, even in the case of the downlink configuration, the system was well within the limit given in the IEEE 802.11g standard for 64-QAM transmission (i.e. it was always much lower than $-25 \mathrm{~dB}$ ) for all luminance levels. Namely, we observed a working system at a luminance value even lower than 15 lux: this was obtained at $3 \mathrm{~m}$, which is a common distance from the ceiling in indoor communications.

\section{Conclusion}

We experimentally demonstrated for the first time a hybrid RoF-VLC system for indoor broadband wireless access. We showed the feasible integration of RoF and VLC technology, using well-established Wi-Fi protocol IEEE 802.11g, and commercially available components. This hybrid RoF-VLC system was used to successfully transmit $54 \mathrm{Mbit} / \mathrm{s}$ over a realistic distance range (1 km SMF, $3 \mathrm{~m}$ free space) and power values. The integrated hybrid RoFVLC system is suitable for bidirectional transmission and its performance is well in accordance with standard specifications of WiFi. Particularly, for the VLC sub-link the luminance level at the Rx is 15 lux, i.e. much lower than recommended for indoor office environment.

\section{Acknowledgements}

The authors would like to thank Dr P. Faccin (Commscope, Italy) for enlightening discussions.

\section{References}

[1] S. Miyauchi, T.Komine, S.Haruyama, and M.Nakagawa, "Analyses of LED allocation algorithm for parallel wireless optical communication", Proc of IEEE Radio and wireless symposium 2006 (RWS2006), pp. 191-194.

[2] H. Elgala et al.,"Indoor broadcasting via white LEDs and OFDM", IEEE Transactions on consumer electronics, vol. 55, no .3, pp. 11271134, August 2009.

[3] T .Komine, M. Nakagawa,. “Fundamental analysis for visible-light communication system using LED lights” IEEE Transactions on Consumer Electronics, Vol. 50, No. 1, pp.100-107, February 2004.

[4] J. Grubor, S. Randel, K. D. Langer, and J. W. Walewski, "Broadband Information Broadcasting Using LED-Based Interior Lighting" Journal of Lightwave Technology, vol. 26, no. 24, December 15, 2008 pp.3883-3892.

[5] N. Shrestha, M. Sohail, C. Viphavakit., P. Saengudomlert, W.S. Mohammed. "Demonstration of visible light communications using RGB LEDs in an indoor environment", in Proc. ECTI-CON 2010, pp.1159-1163.

[6] P. Hartmann, X. Qian, R.V. Penty; I.H. White; "Broadband multimode fibre (MMF) based IEEE 802.1 1a/b/g WLAN distribution system" Microwave Photonics, 2004. 2004 IEEE International Topical Meeting on , vol., no., pp. 173- 176, 4-6 Oct. 2004

[7] H. L. Minh; O'Brien, D. et al., "80 Mbit/s Visible Light Communications using pre-equalized white LED," Optical Communication, 2008. ECOC 2008. 34th European Conference on, vol., no., pp.1-2, 21-25 Sept. 2008.

[8] J. Vučić, L. Fernández, C. Kottke, K. Habel,; K. D. Langer; "Implementation of a real-time DMT-based 100 Mbit/s visible-light link", Optical Communication (ECOC), 2010 36th European Conference and Exhibition on, vol., no., pp.1-5, 19-23 Sept. 2010

[9] European standard EN 12464-1: Lighting of indoor work places, 2003.

[10] IEEE Std. 802.11g, "Further higher-speed physical layer extension in the $2.4 \mathrm{GHz}$ band," 2003. 